\title{
Genetic Variation in the von Willebrand Factor Gene in Swedish von Willebrand Disease Patients
}

\author{
Eric Manderstedt ${ }^{1} \quad$ Christina Lind-Halldén ${ }^{1} \quad$ Stefan Lethagen ${ }^{2,3,4}$ Christer Halldén ${ }^{1}$
}

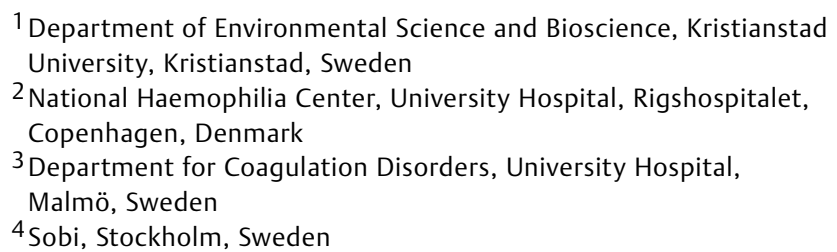

Address for correspondence Eric Manderstedt, MSc, Department of Environmental Science and Bioscience, Kristianstad University, S-23139 Kristianstad, Sweden (e-mail: eric.manderstedt@hkr.se).

TH Open 2018;2:e39-e48.

\begin{abstract}
Keywords

- DNA

- molecular

- diagnosis

- Sweden

- von Willebrand disease

- von Willebrand factor

von Willebrand factor (VWF) level and function are influenced by genetic variation in VWF and several other genes in von Willebrand disease type 1 (VWD1) patients. This study comprehensively screened for VWF variants and investigated the presence of $A B O$ genotypes and common and rare VWF variants in Swedish VWD1 patients. The VWF gene was resequenced using lon Torrent and Sanger sequencing in 126 index cases historically diagnosed with VWD. Exon 7 of the $A B O$ gene was resequenced using Sanger sequencing. Multiplex ligation-dependent probe amplification analysis was used to investigate for copy number variants. Genotyping of 98 single nucleotide variants allowed allele frequency comparisons with public databases. Seven VWD2 mutations and 36 candidate VWD1 mutations ( 5 deletions, 4 nonsense, 21 missense, 1 splice, and 5 synonymous mutations) were identified. Nine mutations were found in more than one family and nine VWD1 index cases carried more than one candidate mutation. The T-allele of rs 1063857 (c.2385T > C, p.Y795 =) and blood group O were both frequent findings and contributed to disease in the Swedish VWD1 population. VWD2 mutations were found in 20 and candidate VWD1 mutations in 51 index cases out of 106 (48\%). VWF mutations, a VWF haplotype, and blood group $\mathrm{O}$ all contributed to explain disease in Swedish VWD1 patients.
\end{abstract}

\section{Introduction}

von Willebrand disease (VWD) is often caused by genetic defects in the von Willebrand factor (VWF) gene and is divided into three major subtypes: type 3 (VWD3) is characterized by total absence of VWF, type 2 (VWD2) by functional disturbance of VWF, and type 1 (VWD1) by low plasma concentration of functionally normal VWF. ${ }^{1}$ Diagnosis of VWD is challenging due to the heterogeneity of the disease and can be made based on phenotypic testing alone; therefore, mutation analysis has not been regarded as necessary. ${ }^{2}$

The highly polymorphic VWF gene spans $178 \mathrm{~kb}$, is composed of 52 exons that vary in size from $50 \mathrm{bp}$ to $1.3 \mathrm{~kb}$, and gives rise to an mRNA that is $\sim 9 \mathrm{~kb}$ in size. There is an unprocessed pseudogene (VWFP1) located on chromosome 22 with $97 \%$ sequence homology to the VWF gene sequence for exons 23 to 34 . The large size, the highly polymorphic sequence, and the presence of the pseudogene complicate screening for pathogenic variants in VWF. VWF mutations have been reviewed, ${ }^{1}$ and they are compiled in the von Willebrand factor Variant Database (http://www.vwf.group.shef.ac.uk/) containing $>700$ unique variants. VWD1 mutations are mostly missense mutations that are spread throughout the whole VWF gene and many show incomplete penetrance. Previous mutation screening in VWD1 identified VWF mutations in 105 of $150(70 \%)$ patients, but 57 of these patients had abnormal received

August 31, 2017 accepted after revision

November 23, 2017
DOI https://doi.org/

10.1055/s-0037-1618571. ISSN 2512-9465. (c) 2018 Georg Thieme Verlag KG
Stuttgart · New York

License terms

(1)(1) 
multimers and VWD2, leaving 93 VWD1 patients with qualitatively normal multimers. ${ }^{3}$ In these patients fewer mutations were identified ( 51 of $93,55 \%$ ). In a similar study, 150 Canadian VWD patients were analyzed for mutations in the VWF gene. ${ }^{4}$ The authors identified putative mutations within the VWF gene in 78 patients (63\%) with qualitatively normal multimers. In both studies, the contributions of other factors such as $A B O$ blood group were more important in milder cases and patients with lower VWF levels had a higher probability of carrying mutations in the VWF gene itself. The mutations act through two major mechanisms: intracellular retention ${ }^{5}$ and rapid clearance; ${ }^{6}$ however, the molecular mechanisms of a substantial number of candidate mutations are still unknown, creating an uncertainty in the classification of VWD1 mutations. Investigating the genetic variation of the VWF gene in healthy controls of African American descent identified several missense variants that were associated with VWF level variation, but also showed high frequencies of certain rare missense variants previously reported as VWD1 mutations that were not associated with VWF levels. ${ }^{7-9}$ This emphasizes the challenge in determining the pathogenicity of candidate VWF mutations.

Meta-analysis of several genome-wide association studies $^{10}$ identified single nucleotide variants (SNVs) in the VWF locus that were significantly associated with VWF level variation. The most significant of these was rs1063857 $\left(p=1.7 \times 10^{-32}\right.$, c.2385T $>$ C, p.Y795 $\left.=\right)$ ) located in a $\sim 30 \mathrm{~kb}$ region defined by 47 significant SNVs. It also identified genes other than VWF that contribute to plasma level variation of VWF in the normal population. ${ }^{10}$ Comparing the effects of these different genetic factors on level of VWF, the $A B O$ blood group was the largest contributor explaining $\sim 25 \%$ of $\mathrm{VWF}$ level variation with much smaller contributions from the other factors including the $V W F$ gene. ${ }^{10}$ According to Johnsen et al, ${ }^{8}$ rare $V W F$ missense variants in aggregate explained 3.3\% of the VWF phenotypic variance, whereas the common VWF variants reported by Campos et $\mathrm{al}^{11}$ explained 0.9 to $1.5 \%$. Since the previously identified common variants explained only a small fraction of the VWF level variation, several normal populations were reanalyzed with Human Exome Bead Chips aimed to investigate rarer coding variants. ${ }^{12}$ VWF levels were affected by a single rare variant in STAB2 and by multiple rare or lowfrequency variants within $A B O$ and surrounding genes.

This study aims to identify candidate $V W F$ mutations in a population of Swedish patients clinically diagnosed with VWD and simultaneously define VWD1 patients with and without VWF mutations. In addition, blood group $\mathrm{O}$ status and the potential influence of common VWF variants on VWD1 status will be investigated.

\section{Materials and Methods}

\section{Study Populations and von Willebrand Disease Phenotyping}

The VWD study population was recruited at the Department for Coagulation Disorders, Malmö University Hospital (Malmö, Sweden). The population consisted of consecutive patients and their relatives who attended the clinic between the years 1988 and 2005 and corresponds to $\sim 1,000$ indivi- duals belonging to 126 families. This population represents the majority of all families diagnosed with VWD in Sweden during this time period. Approximately $50 \%$ of all families were represented by a single index case; the remaining families were represented by 2 to 15 family members. Complete mutation analysis was initially performed on one index case from each of the 126 families. In a second step, the analysis was extended to a total of 288 individuals recruited unevenly from the families. The coagulation department in Malmö is a national referral center for bleeding disorders, and all patients' details are collected in a register. Clinical and laboratory data were recorded for each patient and their bleeding phenotypes were classified. ${ }^{13}$ We used historical VWF levels usually determined at the time of the original diagnostic work-up. Therefore, different phenotypical methods have been used. VWF activity was measured using the traditional VWF: ristocetin cofactor (VWF:RCo) method based on aggregation of platelets or an automated VWF:RCo assay based on the BCS coagulation analyzer using the $\mathrm{BC}$ von Willebrand reagent (Dade Behring Inc., Newark, Delaware, United States). VWF antigen (VWF:Ag) levels were measured with electroimmunoassay (the Laurell's method) and immunoradiometric assay, enzyme-linked immunosorbent assay, or line immunoassay. Multimer analysis and ristocetin-induced platelet aggregation were only performed occasionally. There were no further analyses of VWF levels in this study and the historical VWF levels available do not allow further analyses of VWF levels. In a strict sense, not all index cases fulfilled the diagnostic criteria according to ISTH, ${ }^{14}$ but at the time of diagnosis, their bleeding symptoms in combination with lowered VWF levels were interpreted as being VWD1. A total of 29 of these families have previously been analyzed for cosegregation between polymorphisms in the VWF gene and VWD. ${ }^{13}$ The analyses of the populations were approved by the Ethics Committee of the Medical Faculty, Lund University, and the Swedish Data Inspection Board. Written informed consent was obtained from all subjects.

\section{Ion Torrent Sequencing}

Genomic DNA was isolated from whole blood using QIAamp DNA Mini or Maxi Kits (Qiagen GmbH, Hilden, Germany). The DNA concentrations were determined by fluorometry using PicoGreen (Molecular Probes; Invitrogen, Eugene, Oregon, United States) and all samples were normalized to the same concentration. The Ion Torrent sequencing system (Life Technologies, Carlsbad, California, United States) was used for mutation screening. The primer sets were designed using Ion AmpliSeq Designer (http://www.ampliseq.com, pipeline version 2.2.1). A total of 79 primer pairs that generated amplicons covering $1 \mathrm{~kb}$ promoter and $98 \%$ of the coding sequence of $V W F$ were obtained (-Supplementary Table S1). The homologous regions of VWF and its pseudogene VWFP1 were compared using Ensembl BLAST (http://www.ensembl. org/Multi/blastview) to ascertain that the primers could differentiate between the two genes. The DNA samples were individually barcoded using Ion Xpress Barcode Adapters. Initial amplification of the targeted regions was 
performed using the Ion AmpliSeq Library Kit 2.0. Emulsion polymerase chain reaction (PCR) was performed on the OneTouch 2 system with the Ion PGM Template Hi-Q View OT2 kit. Sequencing was performed on an Ion Torrent PGM using Ion PGM Hi-Q View Sequencing kit and Ion PGM 316 chip v2 according to the manufacturer's instructions (Life Technologies). The sequences were aligned against the human reference sequence using Torrent Suite 5.0.5 and variant calling performed using variant calling parameters tuned for AmpliSeq panels. Annotation of the SNVs was accomplished by submitting them to Ensembl Variant Effect Predictor (http://grch37.ensembl.org/Homo_sapiens/Tools/ VEP), Condel 2.0 (http://bg.upf.edu/fannsdb/), MutationTaster (http://www.mutationtaster.org/), Human Splicing Finder (http://www.umd.be/HSF3/), and NNSPLICE V0.9 (http:// www.fruitfly.org/seq_tools/splice.html).

\section{Sanger Sequencing}

Sanger sequencing was used to resequence the complete exons 26 and 28 and confirm all putative mutations discovered with Ion Torrent sequencing. The human genomic sequence was obtained from genomic build GRCh37p13. Primers for the 52 different exons of the VWF gene and exon 7 of the $A B O$ gene (-Supplementary Table S2) were designed using NCBI Primer-BLAST (http://www.ncbi.nlm.nih.gov/tools/primer-blast/index.cgi) and ordered from DNA Technology A/S (Risskov, Denmark). The samples were sequenced using Big Dye Terminator chemistry, v3.1 (Applied Biosystems, Foster City, California, United States) on an ABI 3130xl sequencer. PCR was performed using KAPA Taq HotStart DNA PCR kit (KAPA Biosystems, Cape Town, South Africa). The primary PCR products were purified with ExoSAP-IT PCR Product Cleanup (Affymetrix, Santa Clara, California, United States), unincorporated dye terminators removed by BigDye XTerminator Purification Kit and sequence data assembled and compared using SeqScape (Applied Biosystems).

\section{Single Nucleotide Variant Genotyping}

Genotypes were determined for 98 common SNV markers (95 with minor allele frequencies [MAFs] > 5\%) using the Sequenom MassARRAY MALDI-TOF system (Sequenom Inc., San Diego, California, United States) (-Supplementary Table 53). Assay design was made using the SpectroDESIGNER software (Sequenom Inc.). Primers were obtained from Metabion $\mathrm{GmbH}$ (Martinsried, Germany). The subsequent steps including the Sequenom MassEXTEND reaction were then performed as described by the manufacturer (http://www.sequenom.com/).

\section{Multiplex Ligation-Dependent Probe Amplification Analysis}

Multiplex ligation-dependent probe amplification (MLPA) was used to search for deletions and duplications affecting the complete gene or specific exons. Two MLPA kits that together covered all exons of the VWF gene (P011-VWF, P012-VWF; MRC-Holland, Amsterdam, The Netherlands) were used according to the manufacturer's instructions (http://www.mrc-holland.com). The MLPA analysis used
50 ng of template DNA and the PCR products were separated on an ABI 3130xl automatic sequencer and analyzed using Coffalyser (MRC-Holland).

\section{Genetical and Statistical Analyses}

Variant data for genomic regions was taken from the CEU population of the 1000 Genomes project (http://ftp.1000genomes.ebi.ac.uk/vol1/ftp/release/20110521/). The CEU population consists of 99 Utah residents with northern and western European ancestry, and has been analyzed extensively in both the HapMap and 1000 Genomes projects. For exonic variants, data were compared with data from the European part of the Exome Aggregation Consortium (ExAC; http://exac.broadinstitute.org), which contains data from $\sim 33,000$ European individuals. All variants identified in the VWD population were compared with the information in these databases and in the von Willebrand factor Variant Database (http:// www.vwf.group.shef.ac.uk/) listing all known VWF mutations. Statistical analyses were made using R statistical software. ${ }^{15}$ All SNVs were evaluated for Hardy-Weinberg's equilibrium and then allele frequencies were tested for association with VWD1 using homogeneity tests based on the asymptotic chisquare distribution. Haploview ${ }^{16}$ was used to characterize the linkage disequilibrium pattern in the VWF gene region using data from the CEU population of the 1000 Genomes database. This defined haploblocks, their cosegregation rates, and the underlying haplotypes in each region. Haplotypes of the VWD1 population were constructed with PHASE v2.1. ${ }^{17}$

\section{Results}

All coding exons of the VWF gene were screened for variants using an AmpliSeq strategy and the Ion Torrent platform. This sequencing was performed for all exons except exon 26 on a total of 126 index cases from presumably unrelated Swedish families. Sanger sequencing was used to resequence the complete exons 26 and 28 in all index cases and confirm all mutation candidates. The Ion Torrent sequencing data covered $100 \%$ of the exonic positions except those of exon 26 . The read depth was $>100 X$ in $>95 \%$ of individuals for $>98 \%$ of positions. The Sanger sequence data were generally of high quality with $>95 \%$ of bases having a Phred score of 30 or higher in $>95 \%$ of individuals.

Seven variants are listed explicitly as VWD2 mutations in the von Willebrand factor Variant Database (http://www. vwf.group.shef.ac.uk/). These were found in 20 families (-Table 1, - Fig. 1). One VWD2 mutation was particularly common and was present in more than $50 \%$ of the families (11 out of 20 families). It is interesting to note that one of the VWD2 mutations (p.G1672R) occurred together with a known VWD1 mutation (p.P1413L) in three out of three families, indicating that these families most likely have a common origin and that these two mutations occur on the same haplotype. In the remaining 106 index cases, a total of three large and two small deletions and 39 SNVs with a $\mathrm{MAF}<0.5 \%$ were identified in the coding sequence (-Table 2). There were four nonsense variants identified in a total of 9 index cases and 25 missense variants identified in 
Table 1 Type 2 VWD mutations identified among the 126 index cases

\begin{tabular}{|l|l|l|l|l|l|l|l|l|}
\hline SNP ID & Chr. position $^{\mathrm{a}}$ & Base change & Exon & $\begin{array}{l}\text { Amino acid } \\
\text { change }\end{array}$ & $\begin{array}{l}\text { Index } \\
\text { cases }\end{array}$ & $\begin{array}{l}\text { MAF in } \\
\text { ExAC }^{\mathrm{b}}\end{array}$ & $\begin{array}{l}\text { In silico } \\
\text { prediction }^{\mathrm{c}}\end{array}$ & $\begin{array}{l}\text { VWD type } \\
\text { in database }^{\mathrm{d}}\end{array}$ \\
\hline rs61748466 & 6155892 & $\mathrm{c} .2278 \mathrm{C}>\mathrm{T}$ & 17 & p.R760C & 1 & 0.00002 & 3 & $2 \mathrm{~N}$ \\
\hline rs267607326 & 6132007 & $\mathrm{c} .3437 \mathrm{~A}>\mathrm{C}$ & 26 & p.Y1146C & 1 & 0 & 4 & $2 \mathrm{~A}$ \\
\hline rs61748511 & 6131999 & $\mathrm{c} .3445 \mathrm{~T}>\mathrm{C}$ & 26 & p.C1149R & 1 & 0 & 4 & $2 \mathrm{~A}$ \\
\hline rs61749395 & 6128641 & $\mathrm{c} .3943 \mathrm{C}>\mathrm{T}$ & 28 & p.R1315C & 2 & 0.00002 & 4 & $2 \mathrm{~A} / \mathrm{M}$ \\
\hline rs61750071 & 6128464 & $\mathrm{c} .4120 \mathrm{C}>\mathrm{T}$ & 28 & p.R1374C & 11 & 0.00002 & 4 & $2 \mathrm{~A} / \mathrm{M}$ \\
\hline rs61750100 & 6128067 & $\mathrm{c} .4517 \mathrm{C}>\mathrm{T}$ & 28 & p.S1506L & 1 & 0 & 4 & $2 \mathrm{~A}$ \\
\hline rs61750598 & 6127570 & $\mathrm{c} .5014 \mathrm{C}>\mathrm{A}$ & 28 & p.G1672R & 3 & 0.0007 & 1 & $2 \mathrm{~A}$ \\
\hline
\end{tabular}

Abbreviations: Chr., chromosome; ExAC, Exome Aggregation Consortium; MAF, minor allele frequency; SNP, single nucleotide polymorphism; VWD, von Willebrand disease.

${ }^{a}$ According to GRCh37p13.

${ }^{\mathrm{b}} \mathrm{MAF}$ observed in 33,000 individuals from the non-Finnish European population in the ExAC database.

${ }^{c}$ For missense mutations, the predictions are classified according to damaging ( 1 ) or tolerated (0) using the programs SIFT, PolyPhen-2, Condel 2.0, and MutationTaster. The table presents the sum of all predictions.

${ }^{d}$ von Willebrand factor Variant Database, previously ISTH-SSC VWF Online Database.

a total of 41 index cases. In addition, there were nine synonymous variants in a total of 15 index cases and one variant potentially affecting splicing in 1 index case. A total of three large and two small deletions were detected by Ion Torrent sequencing. MLPA analysis confirmed the three large

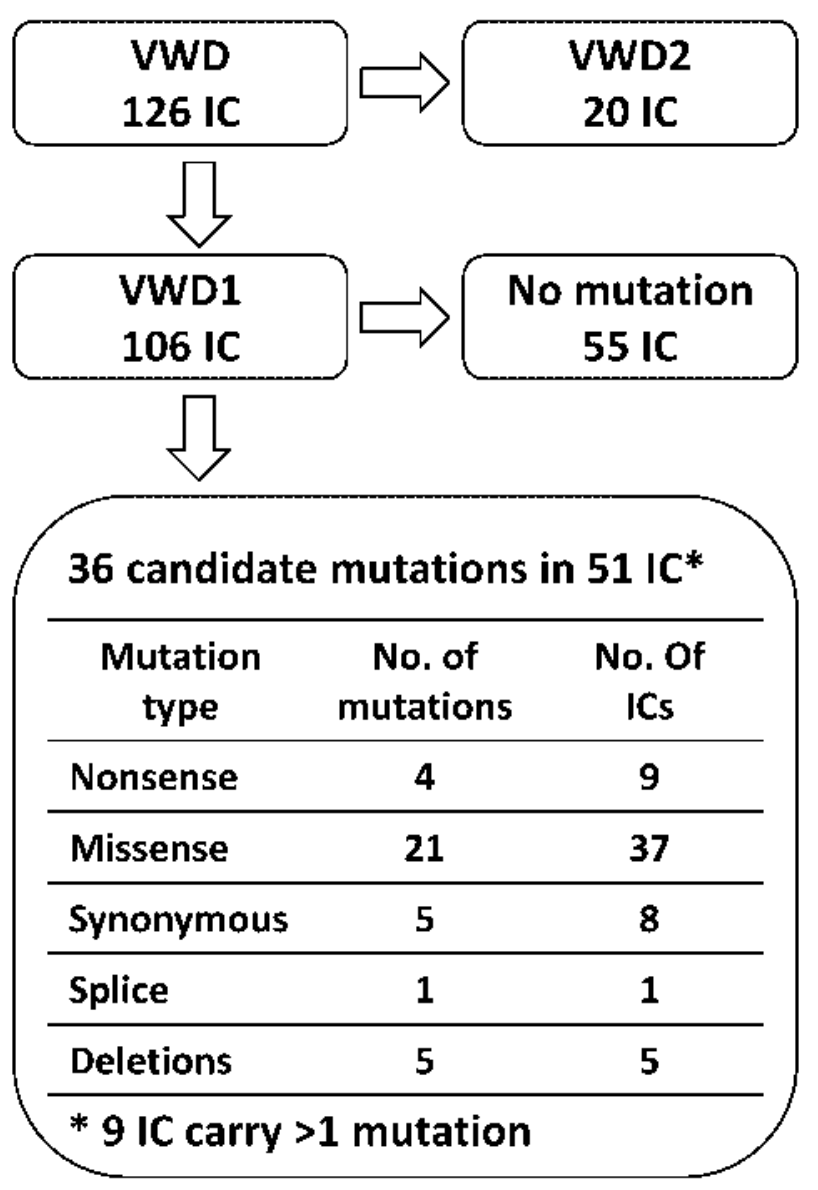

Fig. 1 The mutation status of the 126 von Willebrand disease (VWD) index cases (IC). deletions; one encompassing exons 14 to 52 and two with partial deletions of exon 28 . All were present in one index case each.

Two of the nonsense variants are annotated as VWD3 mutations in the von Willebrand factor Variant Database and these were present in three (p.R2535*) and four (p.R1659*) index cases, respectively ( $\mathbf{- T a b l e ~} \mathbf{2}, \boldsymbol{-}$ Fig. $\mathbf{1}$ ). The remaining two nonsense variants were not previously described and were found in one index case each. Eleven out of the 25 missense variants are annotated as VWD1 mutations in the von Willebrand factor Variant Database. Out of the remaining 14 missense variants, 10 were classified as damaging by at least three out of four prediction programs (SIFT, PolyPhen2, Condel 2.0, and MutationTaster). For the nine synonymous variants, the potential for inducing alternative splicing was evaluated with Human Splice Finder and NNSPLICE. Five were defined as affecting splicing by both programs, among those were c.4146G > T that have previously been reported by Mufti et $\mathrm{al}^{18}$ as a splicing variant. The p.Y1584C mutation was present in 11 index cases and was thus the most common VWD1 mutation in the Swedish population. The nonsense and missense mutations that were detected in the Swedish population are primarily located in the latter part of the VWF gene, from exon 28 onward (-Table 2). Thirteen index cases carried more than one variant, 11 index cases had two variants, and 2 index cases had three variants ( - Table 3 ). Eight out of these had at least one mutation described in the von Willebrand factor Variant Database (marked in bold in - Table 3).

After identification of index cases with (51) and without (55) candidate mutations as defined earlier ( - Fig. $\mathbf{1}$ ), the historical phenotypic data were used to investigate VWF levels and activities among these two groups of patients. VWF levels are given as IU/dL and the patients with mutations showed in general lower values (VWF:Ag; median 34, minimum 10, and maximum 63) than patients without identified VWF mutations (VWF:Ag; median 42, minimum 


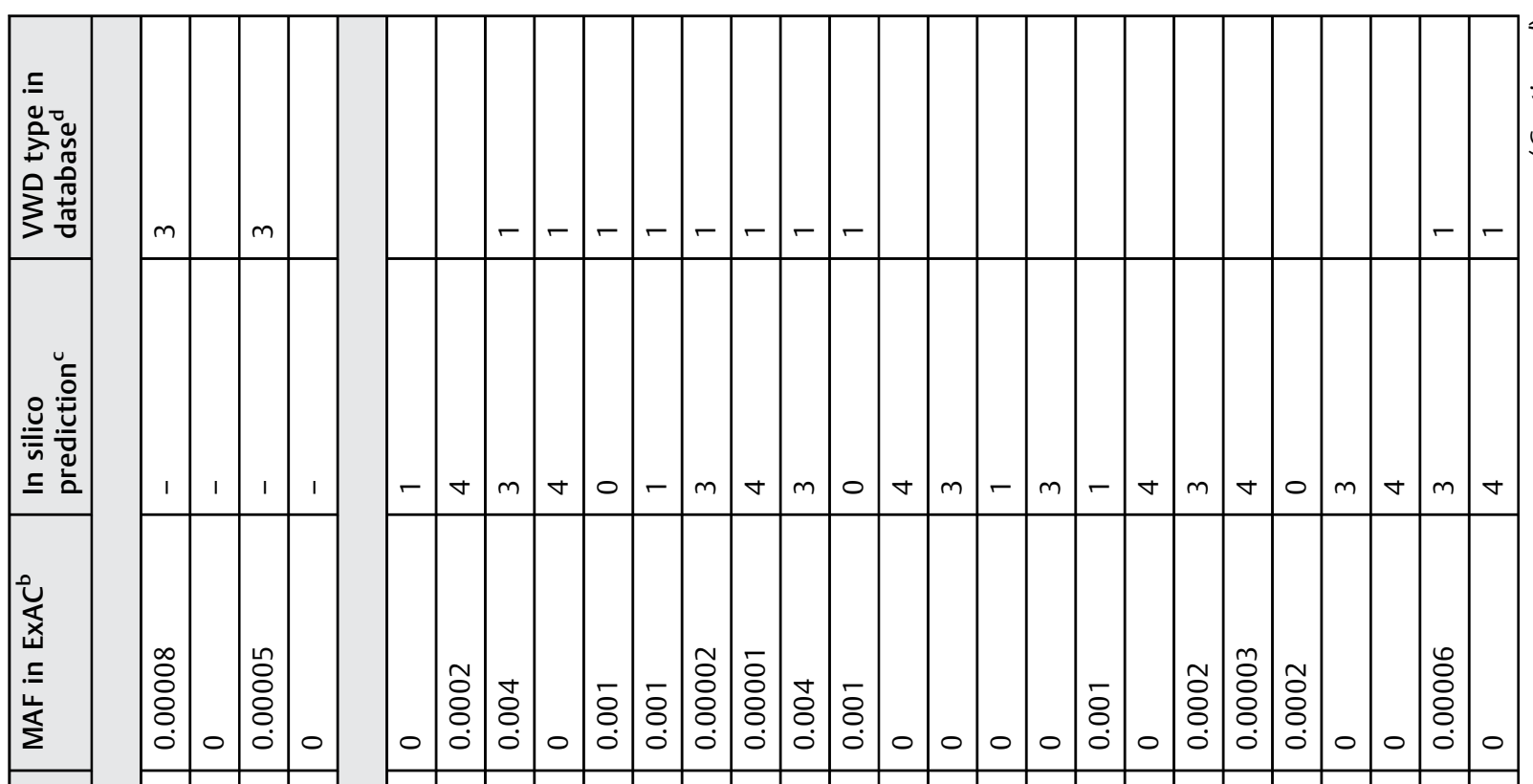

y

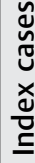

$\checkmark-m-$

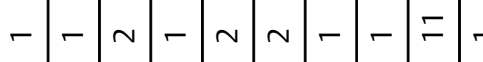

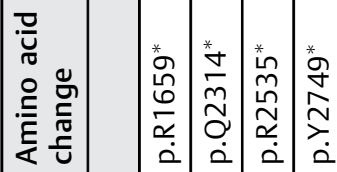

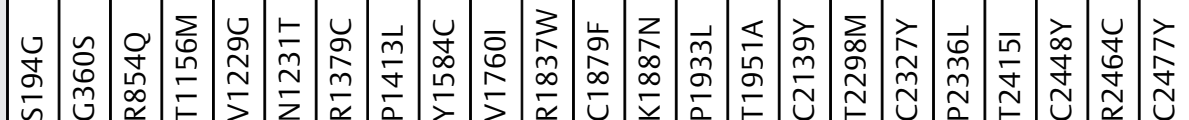

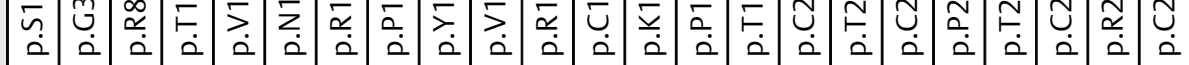

ᄃ̄

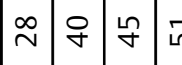

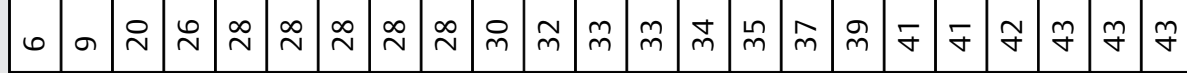

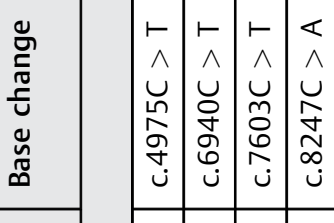

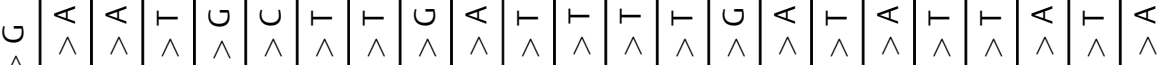
ব́t 管

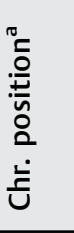

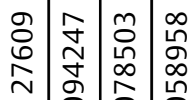

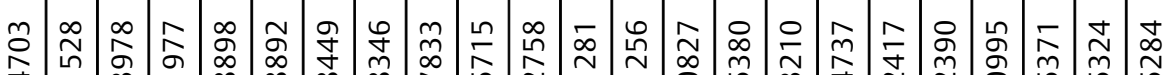

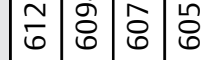

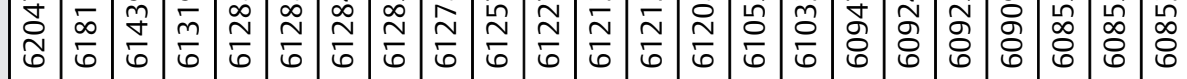

๖

\begin{tabular}{|c|c|c|c|c|c|c|c|c|c|c|c|c|c|c|c|c|c|c|c|c|c|c|c|c|c|c|c|c|c|}
\hline & $\begin{array}{l}\tilde{n} \\
\tilde{\nu} \\
\tilde{\bigsqcup} \\
0\end{array}$ & 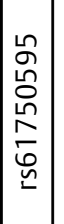 & 竧 & 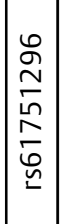 & $\begin{array}{l}3 \\
0 \\
0\end{array}$ & $\mid \begin{array}{l}\dot{n} \\
\tilde{u} \\
\tilde{u} \\
\stackrel{\tilde{\nu}}{\Sigma}\end{array}$ & $\frac{3}{2}$ & 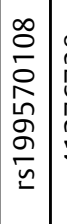 & 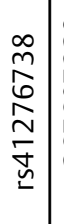 & 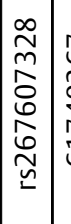 & 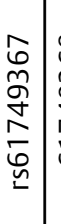 & 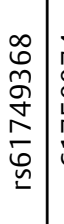 & 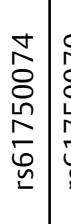 & 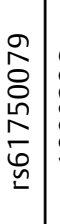 & 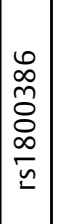 & 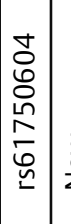 & 23 & 宬 & $\frac{3}{0}$ & $\begin{array}{l}3 \\
0 \\
0\end{array}$ & 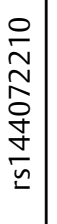 & $\frac{3}{2}$ & $\frac{3}{2}$ & 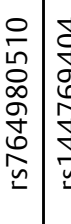 & 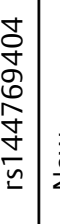 & & zu & 5 & 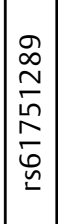 \\
\hline
\end{tabular}




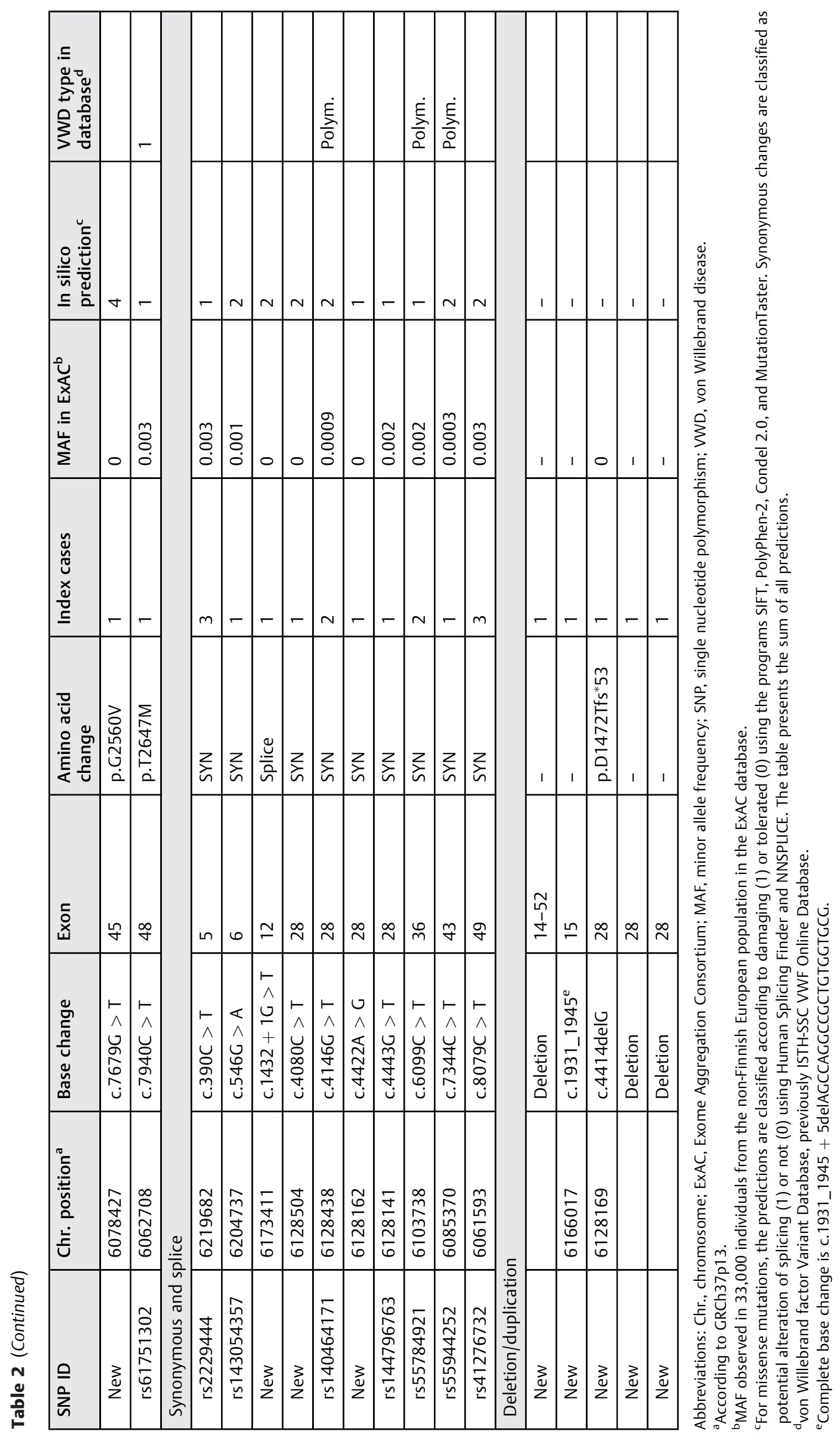


Table 3 Index cases with two or three candidate mutations/variants

\begin{tabular}{|l|l|l|l|l|}
\hline \multirow{2}{*}{ Index case } & \multicolumn{2}{|l|}{ Candidate mutation 1} & \multicolumn{2}{l|}{ Candidate mutation 2 } \\
\cline { 2 - 5 } & Base change & Amino acid change & Base change & Amino acid change \\
\hline 59 & c.8247C $>$ A & p.Y2749* & c.4751A $>$ G & p.Y1584C \\
\hline 46 & c.7603C $>$ T & p.R2535* & c.8079C $>$ T & SYN \\
\hline 87 & c.6980G $>$ A & p.C2327Y & c.7679G $>$ T & p.G2560V \\
\hline $57^{\text {a }}$ & c.6940C $>$ T & p.Q2314* & p.S194G \\
\hline 33 & c.6893C $>$ T & p.T2298M & c.580A $>$ G & SYN \\
\hline 16 & c.5851A $>$ G & p.T1951A & c.546G $>$ A & SYN \\
\hline 64 & c.5636G $>$ T & p.C1879F & c.8079C $>$ T & SYN \\
\hline 56 & c.5509C $>$ T & p.R1837W & c.390C $>$ T & SYN \\
\hline $36^{\text {b }}$ & c.4975C $>$ T & p.R1659* & c.2561G $>$ A & p.R854Q \\
\hline 93 & c.4751A $>$ G & p.Y1584C & c.7244C $>$ T & p.T2415I \\
\hline 38 & c.4751A $>$ G & p.Y1584C & c.390C $>$ T & SYN \\
\hline 73 & c.3686T $>$ G & p.V1229G & c.3692A $>$ C & p.N1231T \\
\hline 5 & c.3686T $>$ G & p.V1229G & c.3692A $>$ C & p.N1231T \\
\hline
\end{tabular}

${ }^{\mathrm{a}}$ Also carries the variant c.390C > T (SYN).

${ }^{\mathrm{b}}$ Also carries the variant c.4146G > T (SYN).

27, and maximum 59). The corresponding VWF activity values were (VWF: RCo; median 37, minimum $<4$, and maximum 71) for patients with mutations and (VWF:RCo; median 48 , minimum 29 , and maximum 56 ) for patients without mutations.

The mutation analysis was extended to include a total of 288 individuals from the 126 families. This was made to allow cosegregation analysis of the candidate mutations with disease in families where this was possible. Complete cosegregation was observed for all type 2 VWD families that could be evaluated. In contrast, incomplete cosegregation was observed for 4 out of 17 investigated type 1 VWD families. However, this analysis is difficult to interpret due to the low and varying number of individuals analyzed in the different families.

Association analysis comparing the 106 VWD1 patients with the CEU population of the 1000 Genomes database using 98 common VWF SNVs (95 with MAF > 5\%) was made to search for SNVs associated with VWD1 status. Only one SNV showed a nominally significant allele frequency difference (rs6489695, $p=0.03$ ). This SNV was not significant after Bonferroni's correction and was located $\sim 100 \mathrm{~kb}$ from rs1063857(c.2385T > C, p.Y795 =) that was identified as the most significant SNV in previous studies. ${ }^{10,11}$ The MAF for rs1063857 among VWD1 patients was 30\%, whereas it was 35\% in the CEU population (-Supplementary Table 53). The major T-allele previously associated with lower VWF levels is thus also enriched in the Swedish VWD1 population. ${ }^{10,11}$

Blood group $\mathrm{O}$ is known to be overrepresented among VWD1 cases, and in this study, 75 index cases (71\% of 106 VWD1 index cases) were of type 0 , clearly higher than the Swedish national average of $\sim 38 \%$ type 0 . Comparing index cases with or without a putative VWF mutation, the type 0 frequency is slightly higher among the mutation-negative cases ( $40 / 55 \geq 73$ vs. $35 / 51 \geq 67 \%$ ) but since the total number of cases is rather low, the observed difference is not significant (-Table 4). As expected, a higher VWF:Ag level was correlated with the absence of a candidate mutation and with blood group O. Comparable with other studies, no overrepresentation of type $O$ was found in index cases carrying a VWD2 mutation (25\%).

The VWD bleeding phenotype is influenced by multiple factors; rare VWF mutations with small or large phenotypic effects, common VWF variants with small effects, blood group $\mathrm{O}$, and several other genes. Combining the information from our VWF mutation analysis with genotyping data of rs1063857 in the VWF gene and blood group O, data show that 58 out of 106 index cases in the Swedish VWD1 population have two or more of these factors that have been associated with low VWF levels (-Fig. 2). The different factors seem to be distributed randomly among the index cases. This observed independence between factors is also

Table 4 VWF:Ag levels among Type 1 VWD patients

\begin{tabular}{|l|l|l|l|}
\hline $\begin{array}{l}\text { Mutation and } \\
\text { blood group } \\
\text { status }\end{array}$ & $\begin{array}{l}\text { VWF:Ag } \\
<30 \mathrm{IU} / \mathrm{dL}\end{array}$ & $\begin{array}{l}\mathrm{VWF} \mathbf{\mathrm { Ag }} \\
\mathbf{3 0 - 5 0} \mathrm{IU} / \mathrm{dL}\end{array}$ & $\begin{array}{l}\mathrm{VWF}: \mathrm{Ag} \\
>\mathbf{5 0} \mathrm{IU} / \mathrm{dL}\end{array}$ \\
\hline Mutation & 21 & 29 & 1 \\
\hline No mutation & 4 & 43 & 8 \\
\hline Blood group O & 14 & 54 & 7 \\
\hline $\begin{array}{l}\text { Blood group } \\
\text { non-O }\end{array}$ & 11 & 18 & 2 \\
\hline
\end{tabular}

Abbreviations: VWD, von Willebrand disease; VWF:Ag, von Willebrand factor antigen. 


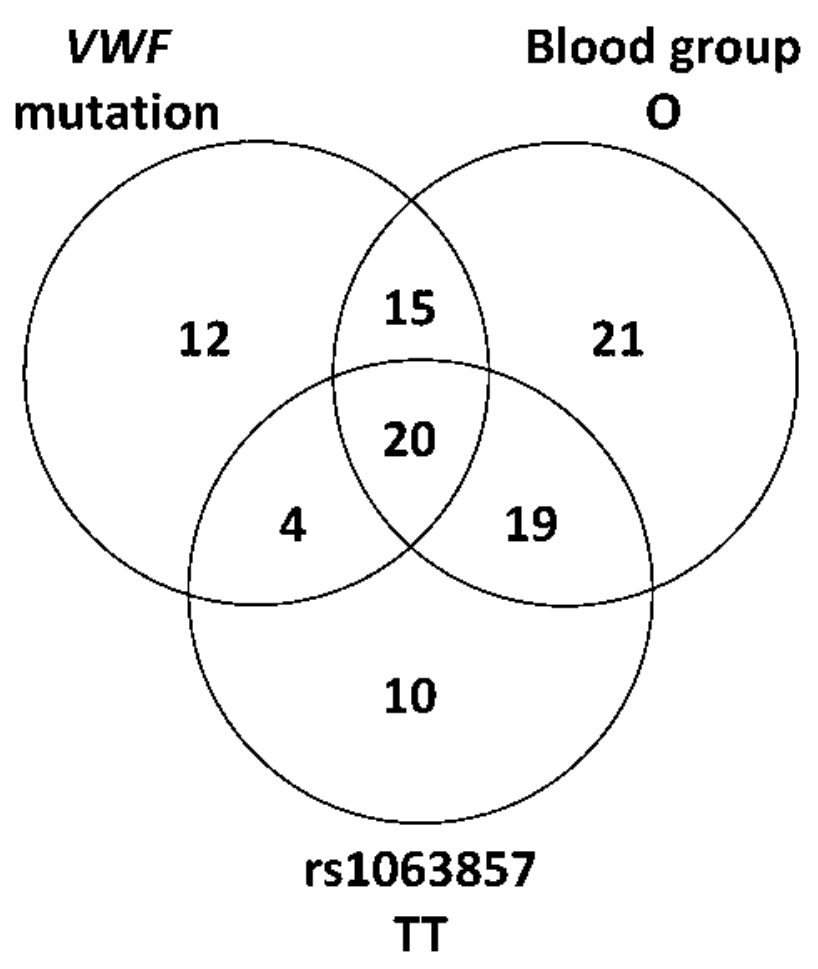

Fig. 2 The presence of von Willebrand factor (VWF) mutations, blood group $\mathrm{O}$, and the TT genotype of rs 1063857 in the 106 von Willebrand disease type 1 index cases.

true for the number of index cases lacking any of the factors ${ }^{5}$ compared with those that have at least one (101).

\section{Discussion}

Several large association studies identified a 30 to $50 \mathrm{~kb}$ region in the VWF gene where several common SNVs were significantly associated with VWF level variation. ${ }^{10,11}$ The most significant SNV in the study by Smith et al ${ }^{10}$ was rs1063857 $\left(p=1.7 \times 10^{-32}\right.$, c.2385T $>$ C, p.Y795 $\left.=\right)$. The effect of this SNV was small, with a one-unit increase in minor allele dosage corresponding to an average increase in the VWF level of $6 \% .^{10}$ The previously disease-associated T-allele was enriched also in the Swedish population compared with the CEU population (70 and 65\%, respectively).

In addition to common variants, there are rare variants that can affect the VWF levels. The contributions of such rare variants can vary in size from the strong effects of bona fide mutations that are fully penetrant and capable of explaining disease on their own, to variants with low impact on phenotype that have low penetrance and must work together with other factors to cause disease. The variants with low impact on phenotype are obviously much more difficult to identify as mutations as they are often not cosegregating with disease in the families where they occur. They are also often present in the background population at higher frequencies in comparison with the bona fide mutations that often appear as private mutations. Thus, neither the presence of candidate mutations in a mutation database nor their absence in a background population is definitive determinants of their eventual effects. More conclusive evidence can in most cases only be achieved through functional studies using cell cultures or various animal models.

This study detected several mutations present in the von Willebrand factor Variant Database: 11 VWD1, 7 VWD2, and 2 VWD3 mutations. The two most common mutations, p.Y1584C (VWD1) and p.R1374C (VWD2), were found in 11 index cases each in our study. The p.Y1584C mutation has been shown to display increased a disintegrin and metalloproteinase with a thrombospondin type 1 motif, member 13 (ADAMTS13) proteolysis, a loss of high-molecular-weight multimers and a lower capacity to support thrombus formation probably as a result of the increased ADAMTS13 cleavage. ${ }^{19}$ This mutation has been found at high frequencies in other studies. It was the most common mutation (15\% of investigated families) in a Canadian cohort study ${ }^{4}$ and in an English study (25\% of families). ${ }^{20}$ It was also found in $8 \%$ of families in a European study. ${ }^{3}$ It has an allele frequency of $0.4 \%$ in the ExAC database and is thus highly enriched in populations selected for VWD. In this study, it was detected in 11 out of 126 families (8.7\%). In eight of these families, 13 out of 14 mutation-carrying individuals had blood group 0 . In the remaining three families, the five mutation-carrying individuals all had non-O blood group, but one had the missense mutation p.T2415I and one had the nonsense mutation p.Y2749* that likely contributed to explain the disease phenotype in those cases. Thus, the effect on VWD of p.Y1584C was in most cases supported by blood group 0 as has been previously reported ${ }^{1,20}$ and in some cases by additional mutations. The p.R1374C mutation is characterized by low VWF level and decreased interaction between VWF and platelet GPIb. Patients carrying this mutation have historically been categorized as VWD1, VWD2A, and VWD2M. ${ }^{21,22}$ It was one of the five most common mutations in a Spanish study ${ }^{23}$ and has also been found in France ${ }^{24}$ and Turkey. ${ }^{25}$ In this study, it was detected in 11 out of 126 families in a total of 31 diseased individuals.

Two nonsense VWD1 mutations occurred in multiple index cases. The p.R1659* mutation was detected in four VWD1 families in heterozygous form in a total of nine patients. All of these patients had blood group 0 . The p. R1659* mutation is annotated in the von Willebrand factor Variant Database (http://www.vwf.group.shef.ac.uk/) as a VWD3 mutation. In heterozygous form, it has previously been reported in VWD1 ${ }^{26}$ and in homozygous form, in VWD3 patients ${ }^{27}$ in Finland and Sweden. It has been reported as fairly common in Finland where it together with another mutation explains the majority of VWD3 in Finland. ${ }^{28}$ It is the most common nonsense mutation in VWF in the ExAC database being present in five individuals. The p.R2535* mutation was detected in three VWD1 families in heterozygous form in a total of five patients and one individual without disease. Three of the patients also had blood group 0 , whereas the two remaining patients and the individual without disease had non-O blood group. This mutation is also annotated in the von Willebrand factor Variant Database (http://www.vwf.group.shef.ac.uk/) as a VWD3 mutation in 
Sweden. ${ }^{26}$ This mutation is the second most common nonsense mutation in the ExAC database and represents the type of recessive mutation that can be fairly common in the general population. These two mutations give rise to a mild phenotype in heterozygous form and can operate in a dominant manner giving rise to VWD1, often together with additional factors such as blood group 0 .

Three missense variants occurred in two index cases each; p.R854Q ${ }^{3}$ p.V1229G, and p.N1231T. ${ }^{4,29}$ These are all annotated as mutations in the von Willebrand factor Variant Database. p.R854Q is a common mutation that has previously been reported in many different studies. Both p.V1229G and p.N1231T occur together on the same haplotype in two index cases. They are the result of a gene conversion event introducing SNVs rs118022194 and rs141852043 of VWFP1 into the VWF gene. One missense variant p.C2327Y occurred in four index cases and is not present in the database, but was predicted to be damaging by all four prediction programs. An additional seven previously reported VWD1 missense mutations were also detected in the Swedish study population: The p.T1156M mutation has previously been reported from Sweden ${ }^{30}$ and Spain. ${ }^{31}$ In vitro coexpression with wild-type and p.T1156M carrying VWF showed a dominant negative effect on secretion. The p.R1379C mutation has been identified in Swedish patients as part of the European MCMDM1VWD study ${ }^{3}$ and in a separate study. ${ }^{32}$ It has also been reported from Spain, ${ }^{31,33}$ but no thorough investigation of its functional consequences has been reported. The p.P1413L and p.V1760I mutations have previously been reported in a European $^{3}$ and a Canadian study. ${ }^{4}$ Cells transfected with the p.R2464C mutation showed a mild reduction in the amount of secreted VWF and characteristic faster running multimeric bands indicating that this mutation is probably causative. Transfections of mutant constructs of p.C2477Y showed intracellular retention and impaired secretion of VWF together with loss of high-molecular-weight multimers. ${ }^{5}$ The p.T2647M mutation has previously been found together with p.S2179F. ${ }^{34}$ Since p.S2179F cosegregated with disease and has been described as causing increased clearance; it is more likely the causal mutation.

There were 20 variants discovered in the VWD1 population that were not present in the dbSNP: two nonsense, 10 missense, 1 splice, 2 synonymous, and 5 deletions. The four nonsense and five deletions are obvious candidates for being true mutations and 8 out of 10 missense variants were classified as damaging by at least three of four prediction programs (SIFT, PolyPhen-2, Condel 2.0, and MutationTaster). In addition, five out of nine synonymous variants were predicted to affect splicing by both prediction programs (Human Splicing Finder and NNSPLICE). One of these five variants, c.4146G $>\mathrm{T}$, has previously been reported to affect splicing. ${ }^{18}$ The 4 nonsense mutations, the 21 missense mutations either annotated as mutations or predicted to be damaging by three or more prediction programs, one splicing mutation, the five synonymous variants predicted to affect splicing by both prediction programs and the three large and two small deletions were present in a total of 51 index cases. Thus, candidate mutations were found in 51 out of 106 index cases (48\%). A recent study in the United States investigated 482 patients historically diagnosed with VWD1. ${ }^{35}$ When these patients were retested, 172 patients did not meet the current diagnostic criteria for VWD1 or low VWF level (VWF:Ag $<50 \mathrm{IU} / \mathrm{dL}$ or $\mathrm{VWF}: \mathrm{RCo}<53 \mathrm{IU} / \mathrm{dL}$ ). There was also no difference in bleeding score whether or not, the current criteria were fulfilled. Complete VWF resequencing showed that $45 \%$ of the 482 patients with historical VWD1 diagnosis carried a rare variant in the VWF gene compared with $62 \%$ of the 310 patients fulfilling the modern criteria. Thus, based on the historical VWD1 diagnosis, their observed mutation discovery frequency (45\%) is directly comparable and highly similar to the mutation discovery rate observed in this study (48\%).

The presence of the pseudogene sequence covering exons 23 to 34 of the VWF gene complicated the screening for mutations in this region. To ascertain that all identified variants in this study were variants in the VWF gene and not the effect of cross-hybridization to the pseudogene sequence, all primer sequences contained at least one mismatch to the pseudogene sequence and all systems were optimized to amplify only the VWF gene sequences. In addition, all amplicon sequences were designed to contain at least one internal position containing a mismatch between the gene and pseudogene sequences. During the sequence interpretation, all sequences were checked for the presence of contaminating pseudogene alleles. The AmpliSeq strategy did not perform well for exon 26 which was instead analyzed using Sanger sequencing. MLPA analysis revealed two exon 28 deletions and one large deletion encompassing exons 14 to 52 . The large deletion has been reported in an earlier study ${ }^{36}$ that evaluated the inheritance of SNV markers.

\section{Authors' Contributions}

E.M. designed the study, performed experiments, interpreted data, and wrote the article. C.L.H. performed experiments and interpreted data. S.L. collected samples and phenotypic data. C.H. designed the study, overviewed the research, interpreted data, and wrote the article.

\section{Conflict of Interest}

None declared.

\section{References}

1 Goodeve AC. The genetic basis of von Willebrand disease. Blood Rev 2010;24(03):123-134

2 De Jong A, Eikenboom J. Developments in the diagnostic procedures for von Willebrand disease. J Thromb Haemost 2016;14 (03):449-460

3 Goodeve A, Eikenboom J, Castaman G, et al. Phenotype and genotype of a cohort of families historically diagnosed with type 1 von Willebrand disease in the European study, Molecular and Clinical Markers for the Diagnosis and Management of Type 1 von Willebrand Disease (MCMDM-1VWD). Blood 2007;109(01):112-121

4 James PD, Notley C, Hegadorn C, et al. The mutational spectrum of type 1 von Willebrand disease: results from a Canadian cohort study. Blood 2007;109(01):145-154

5 Eikenboom J, Hilbert L, Ribba AS, et al. Expression of 14 von Willebrand factor mutations identified in patients with type 1 von Willebrand disease from the MCMDM-1VWD study. J Thromb Haemost 2009;7(08):1304-1312 
6 Haberichter SL, Castaman G, Budde U, et al. Identification of type 1 von Willebrand disease patients with reduced von Willebrand factor survival by assay of the VWF propeptide in the European study: molecular and clinical markers for the diagnosis and management of type 1 VWD (MCMDM-1VWD). Blood 2008;111 (10):4979-4985

7 Bellissimo DB, Christopherson PA, Flood VH, et al. VWF mutations and new sequence variations identified in healthy controls are more frequent in the African-American population. Blood 2012; 119(09):2135-2140

8 Johnsen JM, Auer PL, Morrison AC, et al; NHLBI Exome Sequencing Project. Common and rare von Willebrand factor (VWF) coding variants, VWF levels, and factor VIII levels in African Americans: the NHLBI Exome Sequencing Project. Blood 2013;122(04): 590-597

9 Wang QY, Song J, Gibbs RA, Boerwinkle E, Dong JF, Yu FL. Characterizing polymorphisms and allelic diversity of von Willebrand factor gene in the 1000 Genomes. J Thromb Haemost 2013;11(02):261-269

10 Smith NL, Chen MH, Dehghan A, et al; Wellcome Trust Case Control Consortium. Novel associations of multiple genetic loci with plasma levels of factor VII, factor VIII, and von Willebrand factor: the CHARGE (Cohorts for Heart and Aging Research in Genome Epidemiology) Consortium. Circulation 2010;121(12): 1382-1392

11 Campos M, Sun W, Yu F, et al. Genetic determinants of plasma von Willebrand factor antigen levels: a target gene SNP and haplotype analysis of ARIC cohort. Blood 2011;117(19):5224-5230

12 Huffman JE, de Vries PS, Morrison AC, et al. Rare and lowfrequency variants and their association with plasma levels of fibrinogen, FVII, FVIII, and vWF. Blood 2015;126(11):e19-e29

13 Lanke E, Johansson AM, Halldén C, Lethagen S. Genetic analysis of 31 Swedish type 1 von Willebrand disease families reveals incomplete linkage to the von Willebrand factor gene and a high frequency of a certain disease haplotype. J Thromb Haemost 2005;3(12):2656-2663

14 Sadler JE, Rodeghiero F; ISTH SSC Subcommittee on von Willebrand Factor. Provisional criteria for the diagnosis of VWD type 1. J Thromb Haemost 2005;3(04):775-777

$15 \mathrm{R}$ Development Core Team. R: a language and environment for statistical computing. Vienna, Austria: The R Foundation for Statistical Computing; 2011. Available at: http://www.R-project.org/. Accessed August 18, 2017

16 Barrett JC, Fry B, Maller J, Daly MJ. Haploview: analysis and visualization of LD and haplotype maps. Bioinformatics 2005; 21(02):263-265

17 Stephens M, Donnelly P. A comparison of bayesian methods for haplotype reconstruction from population genotype data. Am J Hum Genet 2003;73(05):1162-1169

18 Mufti AH, Alyami NH, Peake IR, et al. Silent von Willebrand factor variant c.4146G $>$ T (p.Leu1382=) causes type 1 von Willebrand disease via disruption of an exonic splice enhancer motif [abstract]. Res Pract Thromb Haemost 2017;1(Suppl 1):147-148

19 Pruss CM, Golder M, Bryant A, et al. Pathologic mechanisms of type 1 VWD mutations R1205H and Y1584C through in vitro and in vivo mouse models. Blood 2011;117(16):4358-4366

20 Cumming A, Grundy P, Keeney S, et al; UK Haemophilia Centre Doctors' Organisation. An investigation of the von Willebrand factor genotype in UK patients diagnosed to have type 1 von Willebrand disease. Thromb Haemost 2006;96(05):630-641
21 Nitu-Whalley IC, Riddell A, Lee CA, et al. Identification of type 2 von Willebrand disease in previously diagnosed type 1 patients: a reappraisal using phenotypes, genotypes and molecular modelling. Thromb Haemost 2000;84(06):998-1004

22 Penas N, Pérez-Rodríguez A, Torea $\mathrm{JH}$, et al. von Willebrand disease R1374C: type $2 \mathrm{~A}$ or $2 \mathrm{M}$ ? A challenge to the revised classification. High frequency in the northwest of Spain (Galicia). Am J Hematol 2005;80(03):188-196

23 Batlle J, Pérez-Rodríguez A, Corrales I, et al. Molecular and clinical profile of von Willebrand disease in Spain (PCM-EVW-ES): proposal for a new diagnostic paradigm. Thromb Haemost 2016;115(01): 40-50

24 Hilbert L, Gaucher C, Mazurier C. Identification of two mutations (Arg611Cys and Arg611His) in the A1 loop of von Willebrand factor (vWF) responsible for type 2 von Willebrand disease with decreased platelet-dependent function of vWF. Blood 1995;86 (03):1010-1018

25 Berber E, Pehlevan F, Akin M, Capan OY, Kavakli K, Çaglayan SH. A common VWF exon 28 haplotype in the Turkish population. Clin Appl Thromb Hemost 2013;19(05):550-556

26 Zhang ZP, Lindstedt M, Falk G, Blombäck M, Egberg N, Anvret M. Nonsense mutations of the von Willebrand factor gene in patients with von Willebrand disease type III and type I. Am J Hum Genet 1992;51(04):850-858

27 Zhang ZP, Blombäck M, Egberg N, Falk G, Anvret M. Characterization of the von Willebrand factor gene (VWF) in von Willebrand disease type III patients from 24 families of Swedish and Finnish origin. Genomics 1994;21(01):188-193

28 Jokela V, Lassila R, Szanto T, et al. Phenotypic and genotypic characterization of 10 Finnish patients with von Willebrand disease type 3: discovery of two main mutations. Haemophilia 2013;19(06):e344-e348

29 Ahmad F, Jan R, Kannan M, et al. Characterisation of mutations and molecular studies of type 2 von Willebrand disease. Thromb Haemost 2013;109(01):39-46

30 Lethagen S, Isaksson C, Schaedel C, Holmberg L. Von Willebrand's disease caused by compound heterozygosity for a substitution mutation (T1156M) in the D3 domain of the von Willebrand factor and a stop mutation (Q2470X). Thromb Haemost 2002;88(03):421-426

31 Casaña P, Martínez F, Haya S, Espinós C, Aznar JA. Association of the $3467 \mathrm{C}>\mathrm{T}$ mutation (T1156M) in the von Willebrand's factor gene with dominant type 1 von Willebrand's disease. Ann Hematol 2001;80(07):381-383

32 Johansson AM, Halldén C, Säll T, Lethagen S. Variation in the VWF gene in Swedish patients with type 1 von Willebrand Disease. Ann Hum Genet 2011;75(04):447-455

33 Corrales I, Ramírez L, Altisent C, Parra R, Vidal F. Rapid molecular diagnosis of von Willebrand disease by direct sequencing. Detection of 12 novel putative mutations in VWF gene. Thromb Haemost 2009;101(03):570-576

34 James PD, Notley C, Hegadorn C, et al; Association of Hemophilia Clinic Directors of Canada. Challenges in defining type 2M von Willebrand disease: results from a Canadian cohort study. J Thromb Haemost 2007;5(09):1914-1922

35 Flood VH, Christopherson PA, Gill JC, et al. Clinical and laboratory variability in a cohort of patients diagnosed with type 1 VWD in the United States. Blood 2016;127(20):2481-2488

36 Johansson AM, Lanke E, Säll T, Lethagen S, Halldén C. A large deletion identified in a Swedish family with type $1 \mathrm{VWD}$. Thromb Haemost 2011;105(04):733-734 